\title{
On the absence of additional analytic first integrals of the perturbed Barrara problem
}

\author{
Pavel Sevryukov ${ }^{1, *}$ \\ ${ }^{1}$ Stavropol State Pedagogical Institute, 355029, Lenina str., 417a, Stavropol, Russia
}

\begin{abstract}
We consider the problem of additional analytic first integrals within the known problem of the perturbed motion of a satellite in a field determined by the Barrard gravitational potential. The paper demonstrates the absence of additional (different from known) first integrals of the problem.
\end{abstract}

Let us consider the motion of a satellite considered as a material point in the gravitational field of an axisymmetric planet. If the axis of the applicator is directed along the axis of the dynamic symmetry of the planet, and the origin is placed at an arbitrary point of this axis, then the gravitational potential in standard notation is have the form:

$$
U=\frac{f m}{r}\left[1+\sum_{n=1}^{\infty} \frac{I_{n}}{r^{n}} P_{n}\left(\frac{Z}{r}\right)\right]
$$

where $f$ is the gravitational constant, $m$ is the planet mass, $r$ is the radius vector modulus, $I_{n}$ is a constant parameter, $P_{n}$ is the Legendre polynomial of the $\mathrm{n}^{\text {th }}$ order.

The models used to describe the motion of a satellite moving around a compressed axisymmetric planet are fairly well known. The main idea of the known works is the construction of such an approximating expression for the gravitational potential of an axisymmetric planet that would allow integration of the problem in a closed form, in quadratures.

Perhaps the first successful and promising choice of the unperturbed orbit for an artificial Earth satellite was pointed out by J. Vinty [2]. In the particular case for a spheroidal Earth being symmetric relative to the equatorial plane, the same method of constructing the intermediate orbit was elaborated in detail by M. D. Kislikom [8]. Attention is called to the intermediate orbit of R. Barrard [1]. Although for close artificial satellites, it gives a less accurate result than the Vinti problem does, nevertheless, because of its simplicity, it is applicable in constructing the theory of motion of distant satellites of the Earth and, possibly, with great success in the theory of motion of artificial satellites of planets. Proceeding from the classical problem of two fixed centers, E. P. Aksenov, E. A. Grebenikov, and V. G. Demin, as a result of the generalization of the problem mentioned above, clearly demonstrate that the integrable cases of Vinti, Barrara, and Kislik represent

\footnotetext{
* Corresponding author: sevryukovpf@ yandex.ru
} 
particular or limiting cases of a single general problem $[3,4]$. The generalized problem of two fixed centers (sometimes called the Euler problem) is at present the basis of the modern analytical theory of the motion of artificial satellites of the Earth [5, 6].

In papers [10] (in collaboration with A. R. Magomedov) and [11], the author show the non-existence of additional analytic first integrals in the perturbed Euler problem. In this article, the author wants to show the absence of additional analytic first integrals in the Barrard perturbed problem.

The gravitational field of the planet will be approximated by the Barrard gravitational field $[1,6]$. In this case, we place the origin of coordinates in the spherical inertia point of the planet, then $I_{1}=c$. This value is the applicator of the centre of mass of the planet, $I_{2}=0$, and the Barrar potential is to be written as follows:

$$
W=\frac{f m}{r}+\frac{f m}{r^{2}} \sin \varphi,
$$

where $\sin \varphi=\frac{z}{r}$. The remaining terms of the gravitational potential will form the perturbation function:

$$
\begin{gathered}
R=\frac{f m}{r} \sum_{n=3}^{\infty} \frac{I_{n}}{r^{n}} P_{n}(\sin \varphi), \\
\mathrm{U}=\mathrm{W}+\mathrm{R} .
\end{gathered}
$$

The equation of motion of the unperturbed Barrard problem is integrated in closed form in quadratures. The canonical "action-angle" variables were introduced in [7, 12] and expressed in terms of elliptic quadratures. The differential equations of the perturbed Barrard problem take the simplest form if we introduce the canonical variables $L, G, H, l, g$, $h$, like the Delaunay elements of the Keplerian motion, and turn to the corresponding elements for $c=0$. The equations of the perturbed motion in the canonical osculating variables $L, G, H, l, g, h$ will have the form:

$$
\begin{gathered}
\frac{d L}{d t}=\frac{\partial H^{*}}{\partial l}, \quad \frac{d G}{d t}=\frac{\partial H^{*}}{\partial g}, \quad \frac{d H}{d t}=\frac{\partial H^{*}}{\partial h}, \\
\frac{d l}{d t}=-\frac{\partial H^{*}}{\partial L}, \quad \frac{d g}{d t}=-\frac{\partial H^{*}}{\partial G}, \quad \frac{d h}{d t}=-\frac{\partial H^{*}}{\partial H},
\end{gathered}
$$

at that

$$
H^{*}=H_{0}+R
$$

It is clear that in the formula (6) $H_{0}=H_{0}=(L, G, H)$ is the unperturbed Hamiltonian of the Barrard problem, $\mathrm{R}$ is the perturbation function (3), which, taking into account the relations

$$
\begin{aligned}
& \sin \varphi=\sin i \cdot \cos \theta, \\
& \mathrm{r}=\mathrm{p} \frac{1}{1+e \cos v}
\end{aligned}
$$


can be represented in the form

$$
R=f m \sum_{n=3}^{\infty} \frac{I_{n}}{r^{n+1}}\left(\frac{p}{r}\right)^{n+1} P_{n}(\sin i \cos \theta),
$$

In the above formulas, the focal parameter of the satellite's orbit $p=a\left(1-e^{2}\right)$, the latitude argument $\theta=v+\omega ; a$ is the semimajor axis, $e$ is the eccentricity, $i$ is the inclination of the orbit, $v$ is the true anomaly, $\omega$ is the pericentre argument.

We introduce the functions of inclination and eccentricity for the Barrard problem [13, 14]:

$$
\begin{gathered}
L_{n}^{(\gamma)}(i)=\frac{1}{2 \pi} \int_{0}^{2 \pi} P_{n}(\sin i \cos \theta) \cos \gamma \theta d \theta, \\
M_{v}^{(k)}(e)=\frac{1}{2 \pi} \int_{0}^{2 \pi} \frac{\cos k v}{(1+e \cos v)} d v,
\end{gathered}
$$

then the perturbation function can be written in the form

$$
R=f m \sum_{n=3}^{\infty} \sum_{i, \gamma} \frac{I_{n}}{r^{n+1}} M_{-(n+1)}^{(j-\gamma)} L_{n}^{(\gamma)} \exp (\sqrt{-1}(j v+\gamma \omega)) .
$$

The eccentricity functions are related to the Hansen coefficients with zero superscript by the relation

$$
X_{v, k}^{(0)}=\left(1-e^{2}\right)^{v+\frac{3}{2}} M_{v+2}^{(k)}
$$

The slope functions are expressed in terms of the adjoined Legendre functions:

$$
L_{n}^{(g)}=\frac{(n-g) !}{(n+g) !} P_{n}^{(g)}(0) P_{n}^{(g)}(\cos i)
$$

By choosing $\mu=c r_{0}{ }^{-1}, 10^{-6}$, as the small parameter, where $r_{0}$ is the average radius of the planet, we represent the perturbation function by a number

$$
R=\sum_{i \geq 1} \mu^{i} H_{i_{*}}^{*}
$$

Using the coupling formulas given in [6], we express the orbital elements $a, e, i$ in terms of the action variables $L, G, H$. For angular variables, to within $\varepsilon^{2}, v=1$ and $\omega=\mathrm{g}$. Here $e=\frac{c}{p}$. Thus, each function $H_{i}^{*}$ that can be expressed in terms of the variables $L, G, H, l, g$ is periodic in the angular variables $l$ and $g$ with the period of $2 \pi$ :

$$
H_{i}^{*}=\sum_{i, \gamma} A_{j, \gamma}^{i}(L, G, H) \exp (\sqrt{-1}(j l+\gamma g)) \text {. }
$$


In accordance with [6], the unperturbed Hamiltonian of the problem has the form

$$
H_{0}(L, G, H)=\frac{1}{2}\left(\frac{f m}{L}\right)^{2}\left(1+\frac{2 H^{2}}{L G} \varepsilon^{2}+\cdots\right)
$$

It is easy to see that the angular variable $h$ is cyclic; therefore, the Hamiltonian equations (5) admit the first integral

$$
\mathrm{H}=\Lambda=\text { const, }
$$

which makes it possible to lower the order of the original system of equations and obtain the above system

$$
\begin{gathered}
\frac{d L}{d t}=\frac{\partial H^{*}}{\partial l}, \quad \frac{d G}{d t}=\frac{\partial H^{*}}{\partial g}, \\
\frac{d l}{d t}=-\frac{\partial H^{*}}{\partial L}, \quad \frac{d g}{d t}=-\frac{\partial H^{*}}{\partial G},
\end{gathered}
$$

with the Hamiltonian

$$
H^{*}=H_{0}(L, G)+\sum_{i \geq 1} \mu^{i} H_{i}^{*}(L, G, l, g)
$$

In the "New Methods of Celestial Mechanics" by A. Poincare [9], a theorem is proved which, in our case, can be formulated as follows:

Let the motion of the satellite be described by the reduced system (19), and the Hamiltonian has the form (20). Then, if (a) the function $H_{0}$ does not depend on the angular variables $l$ and $g ;(b)$ the Hessian of the function $H_{0}$ with respect to the variables $L$ and $G$ is not zero identically; (c) the functions $H_{i}$ are periodic functions of $l$ and $g$ with the period of $2 \pi$, then the reduced system of equations does not admit any other independent analytic first integrals except for the energy integral $H=$ const for the sufficiently small parameter $\mu$.

It is easy to verify that all the conditions of the theorem are satisfied. The proof is based on the fact that if there was a single-valued integral in the problem, then in the expansion of the perturbation function into a multiple Fourier series with respect to the angular variables, all the coefficients would have to vanish. A study of the $\mathrm{R}$ decomposition shows that all this is not so. Consequently, we must conclude that the above system of equations (19) cannot have any analytic single-valued integrals that are not a consequence of the energy integral and the cyclic integral (18).

\section{References}

1. R. B. Barrar, Astron. Journ, 66, 1 (1961)

2. J. P. Vinti, Res. Nat. Bur. Standards, 63, 3 (1959)

3. E. P. Aksenov, E. A. Grebenikov, V. G. Demin, Artificial Earth Satellites, 8 (1961)

4. E. P. Aksenov, E. A. Grebenikov, V. G. Demin, the Astronomer. Journal, 40, 2 (1963)

5. E. P. Aksenov, Theory of motion of artificial satellites of the Earth (Nauka, Moscow, 1977)

6. V. G. Demin, Motion of an artificial satellite in an off-center gravitational field (Nauka, Moscow, 1968)

7. A. M. Iskakova, Analytical mechanics of bodies of variable mass (KazSU, AlmaAta, 1982) 
8. M. D. Kislik, Artificial Satellites of the Earth, 4 (1960)

9. A. Poincare, New methods of celestial mechanics. Volume 1 (Nauka, Moscow, 1971)

10. P. F. Sevryukov, A. R. Magomedov, Space Research, 26(2), 317-319 (1988)

11. P. F. Sevryukov, On additional analytic first integrals of the problem of the motion of a satellite of a triaxial planet (Perm State University, Perm, 1990)

12. P. F. Sevryukov, Eurasian Union of Scientists (ESU), 2(23), 58-59 (2016)

13. P. F. Sevryukov, Science and Education Today, 8(7) (2016)

14. P. F. Sevryukov, Science and Education Today, 8(9) (2016) 